\title{
High-Throughput Optimization of Adhesion in Multilayers by Superlayer Gradient
}

\author{
S.Yu. Grachev, C. Cuminatto, E. Søndergård, E. Barthel \\ Surface du Verre et Interfaces CNRS/Saint-Gobain UMR 125, 39 quai Lucien Lefranc, 93303 \\ Aubervilliers, Cedex, France
}

\begin{abstract}
We used thickness gradients for high throughput optimization of adhesion in film stacks. The idea is based on the so-called superlayer test where a top layer under high compression exerts a load onto the lower interfaces and may cause delamination and buckling. Thus, on one hand, the thickness gradient of the superlayer results in the gradient of the load. On the other hand, the adhesion gradient can be realized by changing the thickness of an adhesion enhancer (or an adhesion reducer). When applied in two perpendicular directions (cross-gradient), the gradient of the superlayer in one direction and of the adhesion enhancer in the other, the plane of the sample represents a map where the line of delamination relates the interfacial toughness to the thickness of the enhancer.

In our tests we used Mo superlayers under compressive stress of the order of $\sim 1.5 \mathrm{GPa}$ on a Si wafer with a native oxide. The adhesion reduction was observed with this methodology when Ag layer up to $10 \mathrm{~nm}$ thick was deposited onto the substrate prior to Mo deposition. The delamination occurred at Ag thicknesses starting from $\sim 6 \mathrm{~nm}$. This thickness of Ag corresponds to the islands coalescence and formation of a continuous film which immediately results in adhesion reduction. The other test was performed with a step gradient of Ti enhancer placed under a $10 \mathrm{~nm}$ thick Ag layer in otherwise the same arrangement. A single test showed that $2.8 \AA$ of $\mathrm{Ti}$ was sufficient to improve the adhesion between $\mathrm{Ag}$ and $\mathrm{SiO}_{\mathrm{x}}$ by several times.
\end{abstract}

\section{INTRODUCTION}

Film stacks are widely used in industrial applications such as optical filters, thermal insulators, hard protective coatings, and in microelectronics. One of the requirements for applied use of thin films is their mechanical stability. However, weak interfacial toughness is often responsible for failures of products. In this context, a reliable and easily applicable test of adhesion is a highly wanted tool for mechanical optimization studies.

The superlayer adhesion test has been used both in adhesion estimation and in fundamental research [1-5]. In this test a superlayer under high compression is deposited directly onto a stack. In this case, there is a tendency for the superlayer to relax the internal stress by breaking a weak interface of the stack and buckling. The load on the interface increases with increase of the elastic energy stored in the stressed layer. Since the elastic energy is proportional to the layer thickness $\left(E_{\text {elastic }}=\sigma^{2} h E /(1-v)\right.$; $\sigma$ is the stress, $h$ is the thickness, $E$ is the Young modulus and $v$ is the Poisson's ratio), a gradient of the thickness of the stressed layer provides a gradient of load. When the thickness of the superlayer is large enough, the delamination may occur.

The use of gradients of the superlayer can give a quantitative value of the threshold thickness for delamination. In this way, the adhesion of interfaces can be compared, although the absolute value of the interfacial toughness is difficult to obtain [6]. Using the same gradient method, a gradient of adhesion can also be obtained. Indeed adhesion may depend upon film 
thickness because an interlayer can either promote or reduce adhesion. Here we demonstrate that cross gradient samples can be obtained where a thickness gradients of the superlayer and an adhesion gradient are applied in orthogonal directions simultaneously in one sample. We show that cross-gradients provide a fast test of the impact of an interlayer thickness on adhesion,

\section{EXPERIMENT}

2 inch $(50 \mathrm{~mm})$ wafers of $\mathrm{Si}(100)$ with a native oxide were used as substrates. All the films were obtained by dc sputter-deposition technique at room temperature. A high vacuum deposition chamber with a base pressure of $1 \times 10^{-5} \mathrm{~Pa}$ was used. We applied Mo films under $\sim 1.5$ GPa compressive stress as superlayers. For nominally homogeneous Mo layers the thickness varied by less than 10 \% across the sample, which insured the control over thickness of the films. More details on experimental conditions are given elsewhere [6].

Thickness gradients were obtained by gradually shading the substrate by a metallic shutter during deposition. The plate was moved either continuously with a constant speed or by discrete steps resulting in the continuous gradient or in a series of stripes of homogeneous thickness, respectively. After rotating the sample by $90^{\circ}$ around its normal another gradient was applied (cross-gradient). We realized a gradient of an adhesion modifier ( $\mathrm{Ti}$ or $\mathrm{Ag}$ ) in one direction and a gradient of the Mo superlayer in the perpendicular direction.

\section{RESULTS AND DISCUSSION}

Figure 1 shows a photo of a sample with a continuous cross-gradient of Ag vs. Mo in the stack $\mathrm{Mo} / \mathrm{Ag} / \mathrm{Si}(100)$. The thickness of $\mathrm{Ag}$ is constant along vertical lines and continuously increases from 0 at the lefthand side to $10 \mathrm{~nm}$ at the righthand side of the wafer. The thickness of the Mo superlayer increases from $150 \mathrm{~nm}$ at the bottow, below the horizontal line, to $450 \mathrm{~nm}$ at the upper end of the wafer. The adhesion between Ag and oxides is known to be weak [7]. This is why the addition of Ag reduces the mechanical stability of the stack. At a certain threshold of $\sim 6 \mathrm{~nm}$ of $\mathrm{Ag}$ (the right vertical line) the adhesion became weak enough and delamination and buckling of the layer has occurred. The delamination started in the area with thicker Mo and thicker Ag layers and propagated towards smaller thickness areas before stopping at some threshold thickness which is found to depend upon Ag thickness. Therefore at each thickness of $\mathrm{Ag}$, a threshold thickness of the superlayer can be determined, which reflects the strength of the weak interface or the interfacial toughness. Remarkably, the toughness of the $\mathrm{Ag} / \mathrm{SiO} \mathrm{x}_{\mathrm{x}}$ interface decreased rapidly as the thickness of $\mathrm{Ag}$ reached the critical value of $\sim 6 \mathrm{~nm}$. As the thickness of $\mathrm{Ag}$ increased further, the threshold thickness of the superlayer for delamination decreased to $\sim 200 \mathrm{~nm}$. This must be connected to the fact that at room temperature Ag first forms islands which coalesce and form a continuous film at about 5-8 $\mathrm{nm}$ of $\mathrm{Ag}[8,9]$. After coalescence a continuous $\mathrm{Ag}$ film forms resulting in the loss of direct $\mathrm{Mo} / \mathrm{SiO}_{\mathrm{x}}$ coupling and lower adhesion. 


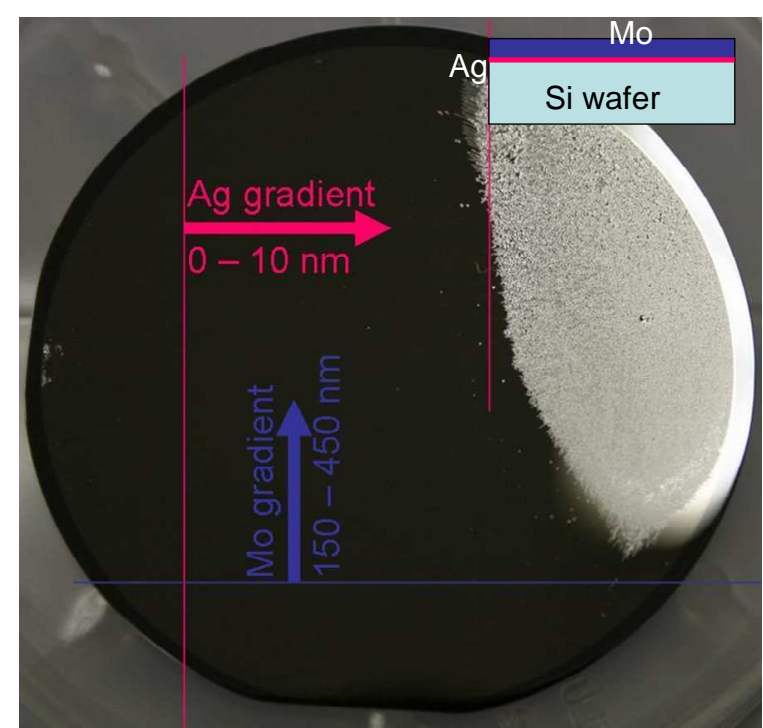

Figure 1. The delamination pattern due to a continuous cross-gradient Ag vs. Mo in a stack $\mathrm{Mo} / \mathrm{Ag} / \mathrm{Si}(100)$. The $\mathrm{Ag}$ thickness was changed from 0 at the left red line to $10 \mathrm{~nm}$ at the right end of the sample. The thickness of Mo superlayer was changed from $150 \mathrm{~nm}$ at the blue line and below to $450 \mathrm{~nm}$ at the upper end of the sample. The buckling of the sample was observed in the area with thicker Mo and thicker Ag layer.

Although the adhesion between $\mathrm{Ag}$ and $\mathrm{SiO}_{\mathrm{x}}$ is weak, it can be improved by adding to this interface an adhesion enhancer, for example, Ti. The effect of Ti is demonstrated in our second experiment. We have prepared a cross-gradient Ti vs. Mo in a stack Mo/Ag(10 $\mathrm{nm}) / \mathrm{Ti} / \mathrm{Si}(100)$. In this experiment, the thicknesses were changed in steps. The thickness of $\mathrm{Ti}$ was $0,1.4,2.8$ and $4.2 \AA$. The thickness of Mo was chosen to be 206, 412, 618 and $824 \mathrm{~nm}$. The delamination pattern is shown in figure 2 .

When no $\mathrm{Ti}$ was present at the $\mathrm{Ag} / \mathrm{SiO}_{\mathrm{x}}$ interface the application of Mo superlayer resulted in delamination all through the Mo thickness. This means that the threshold thickness for delamination was below $206 \mathrm{~nm}$. As $1.4 \AA$ of Ti was added to the weak interface, the threshold thickness had increased to $412 \mathrm{~nm}$. Deposition of $2.8 \AA$ of Ti enhanced the adhesion dramatically and the threshold thickness for delamination elevated to $\sim 824 \mathrm{~nm}$. The adhesion with thicker Ti enhancer was out of range of the superlayer test.

Note that the blisters in both these experiments were of the telephone-cord shape [5]. This shape allows for relaxation of the biaxial stress in both in-plane directions. However the mechanics of the telephone-cord blisters is not well understood and is currently under investigation [5]. In addition to the adhesion optimization, the present tests give access to the morphologies of the telephone-cords for a wide range of thicknesses and adhesions. 


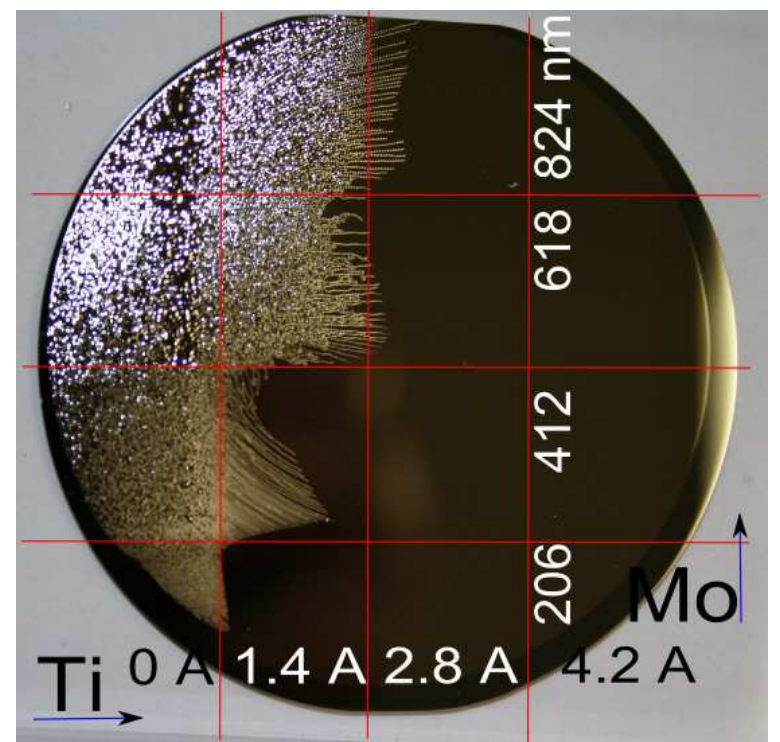

Figure 2. The delamination pattern due to the step cross-gradient Ti vs. Mo in a stack Mo/Ag (10 $\mathrm{nm}) / \mathrm{Ti} / \mathrm{Si}(100)$. The thicknesses are given in the picture. Addition of Ti improved adhesion between $\mathrm{Ag}$ and $\mathrm{Si}(100)$ systematically and the threshold for delamination increased with $\mathrm{Ti}$ thickness.

\section{CONCLUSIONS}

The high throughput approach to the adhesion optimization based on the superlayer test of adhesion has been developed. We used a thickness gradient of Mo superlayer in one direction and a thickness gradient of the adhesion modifier in the perpendicular one. The first gradient provided a range of load onto tested interface and the second one resulted in the gradient of interfacial toughness. This geometry allowed for comparative studies of adhesion versus the thickness of an adhesion modifier.

With this test we found that the adhesion between an $\mathrm{Ag}$ layer and the native silicon oxide on a Si wafer dramatically decreases at $\sim 6 \mathrm{~nm}$ of Ag. At this thickness the Ag islands coalesce and start forming a continuous film. The test was also used with the gradient of $\mathrm{Ti}$ interlayer between $\mathrm{Ag}$ and $\mathrm{SiOx}$. The increase of thickness of Ti resulted in the increased adhesion in this system. A change of Ti thickness with a step of $1.4 \AA$ immediately resulted in the interfacial toughness, which was detected by the superlayer test.

\section{ACKNOWLEDGMENTS}

This project is partly funded by the ANR project MatetPro07_247145.

\section{REFERENCES}

1. Bagchi, G.Lucas, Z.Suo, and A.Evans, J. Mater. Res. 9 (1994) 1734.

2. M.D.Kriese, W.W.Gerberich, and N.R.Moody, J. Mater. Res. 14 (1999) 3007 and 3019.

3. A.Lee, C.S.Litteken, R.H.Dauskardt, and W.D.Nix, Acta Mater. 53 (2005) 609.

4. M.J.Cordill, D.F.Bahr, N.R.Moody, and W.W.Gerberich, Mater. Sci. Eng. A 443 (2007) 150. 
5. C.Coupeau, Mater. Sci. Eng. A 483-484 (2008) 617.

6. S.Yu. Grachev, A. Mehlich, J.-D. Kamminga, E. Barthel, E. Søndergård, Thin Solid Films, submitted.

7. E. Barthel, O Kerjan, P. Nael and N. Nadaud, Thin Solid Films, 473 (2005) 272-7..

8. R. Lazzari, J. Jupille, Surf. Sci. 482-485 (2001), 823.

9. S. Banerjee, S. Kundu, Surf. Sci. 537 (2003), 153. 